\title{
Composição química e avaliações físicas de mortadela de tilápia do Nilo com sabor de camarão
}

\section{Chemical composition and physical evaluations of Nile tilapia bologna with shrimp flavor}

\author{
Raimundo Bernadino Filho ${ }^{1}$, Osvaldo Soares da Silva², Silvana Nazareth de Oliveira ${ }^{3}$, Artur Xavier \\ Mesquita de Queiroga $a^{4}$ Bruno Alexandre de Araújo Sousa ${ }^{5}$.
}

${ }^{1}$ Doutor, Universidade Federal do Agreste de Pernambuco, Garanhuns, Pernambuco, +5587999843810, raimundobfh@gmail.com; ${ }^{2}$ Doutor, Universidade Federal de Campina Grande, Campina Grande, Paraíba, osvaldo_so2002@yahoo.com.br; 3Doutora, Universidade Federal do Agreste de Pernambuco, Garanhuns, Pernambuco, silvana_nazareth@yahoo.com.br; ${ }^{4}$ Doutor, Universidade Federal de Campina Grande, Sousa, Paraíba, arthur.queiroga12@hotmail.com, ${ }^{5}$ Mestre, Instituto Federal da Paraíba, Sousa, Paraíba, baas311@hotmail.com.

\section{A R T I G O}

Recebido: $24 / 03 / 2020$

Aprovado: 26/05/2020

\section{Palavras-chave:}

Emulsionado

Pescado

Crustáceo

Composição centesimal

Key words:

Emulsified

Fish

Crustacean

Centesimal composition

\section{R E S U M O}

As indústrias de beneficiamento do pescado geram grandes quantidades de resíduos que são verdadeiras fontes de contaminação ao meio ambiente quando são descartados inadequadamente. $\mathrm{O}$ aproveitamento desses resíduos na elaboração de alimentos para humanos pode ser uma alternativa na obtenção de um produto nutritivo e mais acessível, além de agregar valor aos resíduos e aumentar a margem de lucro das indústrias. Nesta pesquisa objetivou-se determinar a composição centesimal, valor energético e análises físicas de uma mortadela elaborada com carne mecanicamente separada de tilápia do Nilo com sabor de camarão. Foram analisadas três formulações de mortadelas com concentrações variadas de extrato aromático sabor camarão e realizadas análises de composição química parcial, cálculo do valor energético e análises físicas em equipamentos apropriados para cada análise. Todas as formulações se apresentaram dentro dos padrões estabelecidos pela legislação vigente no Brasil quanto a composição química, além de um valor energético menor que as mortadelas convencionais comercializadas em supermercados. As análises físicas atenderam aos padrões de qualidade recomendados na literatura. As formulações avaliadas são uma alternativa para agregar valor aos resíduos do processamento da filetagem de tilápia, podendo ser empregados na elaboração de mortadela, com características mais saudáveis e nutritivas.

\section{A B S T R A C T}

Sin processing industries generate large amounts of waste that are truly sources of contamination to the environment when they are disposed of inappropriately. The use of these residues in the preparation of food for humans can be an alternative in obtaining a nutritious and more accessible product, in addition to adding value to the residues and increasing the profit margin of the industries. This research aimed to determine the centesimal composition, energy value and physical analysis of a mortadella made with meat mechanically separated from Nile tilapia with shrimp flavor. Three mortadella formulations with varying concentrations of aromatic shrimp flavor were analyzed and partial chemical composition analysis, energy value calculation and physical analysis were performed in appropriate equipment for each analysis. All formulations were within the standards established by current legislation in Brazil regarding chemical composition, in addition to a lower energy value than conventional mortadella sold in supermarkets. The physical analyzes met the quality standards recommended in the literature. The formulations evaluated are an alternative to add value to the waste from the processing of filleting tilapia, and can be used in the preparation of mortadella, with healthier and more nutritious characteristics.

\author{
Revista Verde \\ ISSN 1981-8203 \\ Pombal, Paraíba, Brasil
}

v. 15, n.3, jul.-set., p.250-255, 2020

doi: $10.18378 /$ rvads.v15i3.7772 


\section{INTRODUÇÃO}

Os consumidores estão cada vez mais exigentes por alimentos saudáveis e isso tem motivado a indústria alimentícia a buscar matérias-primas e ingredientes para o desenvolvimento de novos produtos e reformulações dos produtos tradicionais. Esses consumidores buscam alimentos que sejam saborosos, visualmente atrativos e que, ao mesmo tempo, visem à saúde e o bem-estar. As carnes brancas dos peixes vão de encontro a essa nova realidade, pois são ricas em lipídios e proteínas de elevada digestibilidade e qualidade (BERNADINO FILHO et al., 2019)

A tilápia do Nilo (Oreochromis niloticus) é a espécie de peixe que apresenta maiores índices de produção aquícola no Brasil, sendo que os principais produtos comercializados desta espécie são peixes inteiros congelados e, principalmente, os filés que representam a preferência de consumo da carne de tilápia pelo mercado consumidor nacional e internacional (IBGE, 2016). Entretanto, apesar dos diversos aspectos positivos relativos ao cultivo da tilápia, uma das características indesejáveis desta espécie é o baixo rendimento de filé que fica em torno de 32 a 35\%, gerando assim, grandes quantidades de resíduos nas indústrias pesqueiras, diminuindo a margem de lucro do sistema de produção (BARROSO et al., 2017).

Uma forma de aproveitamento dos resíduos sólidos da filetagem de tilápia que vem ganhando espaço no mercado é a obtenção da Carne Mecanicamente Separada - CMS, que pode promover uma valorização e agregação de valor a estes resíduos. A CMS constitui a fração comestível do pescado separada mecanicamente e seu rendimento em carne é superior ao da filetagem. Oferecendo maior vantagem para o produtor e também para o consumidor por ser um produto de alta qualidade nutricional. Esta matéria-prima pode ser utilizada para elaboração de surimi, kamaboko, análogos e embutidos emulsionados, para os quais o mercado está sendo direcionado. (GONÇALVES, 2011).

Potencialmente, três subprodutos podem ser isolados a partir do cefalotórax e casca de camarão, que são os pigmentos carotenoides, quitina/quitosana e extrato saborizante, os quais são de grande utilização na indústria de alimentos e de ração para animais. $\mathrm{O}$ extrato da cabeça de camarão, também denominado de saborizante, pode ser utilizado em uma gama de alimentos, principalmente em produtos à base de Carne Mecanicamente Separada de Pescado (CMSP) ou de surimi, como o fishburguer, kamaboko, chikuwa, embutidos, entre outros alimentos análogos de origem marinha (BASILIO et al., 2003).

Os embutidos têm se destacado como os produtos cárneos de maior produção e consumo no Brasil, indicando que seriam os mais aceitos e os mais acessíveis à população com menor renda (BERNADINO FILHO et al., 2019). O uso da CMS proveniente de peixes de espécies subutilizadas comercialmente ou de resíduos da filetagem, aliado à adição de um extrato aromático obtido dos resíduos do camarão, pode facilitar o acesso da população a um alimento com elevado valor nutricional e com características funcionais, com intuito de fornecer um alimento mais saudável e com preço acessível aos consumidores.
Esta ideia pode ser também uma alternativa para oferecimento de um alimento com sabor diferenciado à base de camarão e de fácil preparo, além de promover maior lucratividade na indústria pesqueira por possibilitar a utilização dos resíduos do processamento de camarão e da tilápia e de evitar o seu descarte no meio ambiente.

Diante do contexto exposto, objetivou-se determinar a composição química parcial, valor energético e análises físicas de uma mortadela elaborada com carne de tilápia adicionada de extrato aromático com sabor de camarão.

\section{MATERIAL E MÉTODOS}

\section{Elaboração das mortadelas}

A matéria-prima utilizada para elaboração das mortadelas foi a Carne Mecanicamente Separada (CMS) obtida de carcaças de tilápia do Nilo da espécie Oreochromis niloticus, provenientes do processo de filetagem manual. Foram desenvolvidas três formulações (Tabela 1) com concentrações variadas de extrato aromático sabor camarão obtidos a partir de resíduos (cefalotórax e carapaça). Os emulsionados foram elaborados baseado na técnica descrita por Moreira et al., (2008), obedecendo o Regulamento Técnico de Qualidade e Identidade de Mortadela (BRASIL, 2000).

Tabela 1. Formulação das mortadelas de tilápia com sabor de camarão

\begin{tabular}{lccc}
\hline & \multicolumn{3}{c}{ Tratamentos } \\
\hline \multirow{2}{*}{ Item } & $\mathrm{M}_{0,5}$ & $\mathrm{M}_{0,75}$ & $\mathrm{M}_{1}$ \\
\cline { 2 - 4 } & $(\%)$ & $(\%)$ & $(\%)$ \\
\hline CMS & 100 & 100 & 100 \\
Saborizante de camarão & 0,5 & 0,75 & 1 \\
\hline Ingredientes de porcentagem fixa & & & \\
\hline Sal & 1,50 & 1,50 & 1,50 \\
Sal de cura & 0,15 & 0,15 & 0,15 \\
Antioxidante & 0,20 & 0,20 & 0,20 \\
Alho em pó & 0,05 & 0,05 & 0,05 \\
Cebola em pó & 0,05 & 0,05 & 0,05 \\
Pimenta branca & 0,05 & 0,05 & 0,05 \\
Condimento para mortadela & 0,05 & 0,05 & 0,05 \\
Corante carmim de cochonilha & 0,07 & 0,07 & 0,07 \\
PTS & 04,0 & 04,0 & 04,0 \\
Gelo & 15,0 & 15,0 & 15,0 \\
\hline
\end{tabular}

Fonte: Adaptado de Moreira et al. (2008)

\section{Análises físico-químicas e valor energético}

A análise de composição química parcial (umidade, cinzas e proteínas) das formulações elaboradas foram determinadas de acordo com as metodologias propostas pela Association of Official Analytical Chemists (AOAC, 2010). O conteúdo de lipídeo total foi determinado utilizando-se o do método de extração de Folch et al. (1957). Os carboidratos foram calculados por diferença de acordo com a Resolução RDC $\mathrm{n}^{\circ}$ 360, de 23 de dezembro de 2003 (BRASIL, 2003). Também foram determinados os teores de cálcio em base seca e amido de acordo com metodologia do Instituto Adolfo Lutz (2008). 
Todas as determinações foram feitas em quatro repetições e o resultado expresso pela média dos valores obtidos.

O valor energético total (VET), em kcal g-1, foi calculado de acordo com a Equação 1:

$$
\mathrm{VET}=(\mathrm{C} \times 4)+(\mathrm{P} \times 4)+(\mathrm{L} \times 9)
$$

Em que, $\mathrm{C}=$ carboidratos, $\mathrm{P}=$ proteínas, $\mathrm{L}=$ lipídios (BUTTE, CABALLERO, 2006).

\section{Análises físicas}

Para a medida da cor foi utilizado colorímetro, com iluminante D65 e ângulo de visão de $10^{\circ}$. Os valores de $\mathrm{L}^{*}$ (luminosidade), $\mathrm{a}^{*}$ (componente vermelho-verde) e $\mathrm{b}^{*}$ (componente amarelo-azul) foram expressos conforme $\mathrm{o}$ sistema de cor da Commission Internationale de L'Eclairage (CIELAB), Minolta (1998). A atividade de água (Aw) foi avaliada a $25{ }^{\circ} \mathrm{C}$ em determinador de atividade de água (4TE, Aqualab). A força de cisalhamento das formulações, foi avaliada com o auxílio de texturômetro TA. HD plus. Os resultados da força mínima necessária para efetuar o corte foram expressos em Kgf. As formulações foram cortadas em pedaços de $2 \mathrm{~cm}$ de altura por $2 \mathrm{~cm}$ de largura. As medidas de $\mathrm{pH}$ foram realizadas sob temperatura ambiente utilizando potenciômetro de acordo com metodologia proposta pelo IAL (2008).

O rendimento de cozimento foi calculado de acordo com a Equação 2, proposta por Horita et al. (2011).

$$
\mathrm{RC}=100 \times \mathrm{P} / \mathrm{P}^{\prime}
$$

Em que, $\mathrm{RC}=$ rendimento de cozimento $\mathrm{P}=$ peso dos embutidos cozidos $\mathrm{P}^{\prime}=$ peso dos embutidos crus.

\section{Oxidação lipídica (TBARS)}

A análise de oxidação lipídica das formulações foi determinada pelo método de substâncias reativas ao ácido tiobarbitúrico (TBARS) de acordo com Vyncke (1970).

\section{Análise Estatística}

Nos dados gerados durante as análises realizadas, foram calculados as médias e os desvios padrões e realizada a Análise de Variância (ANOVA), posteriormente, as médias foram comparadas pelo teste de Tukey, ao nível de 5\% de significância pelo programa software ASSISTAT ${ }^{\circledR}$, versão 7.7 (SILVA et al., 2016).

\section{RESULTADOS E DISCUSSÃO}

\section{Análises físico-químicas e valor energético}

A legislação brasileira determina para as mortadelas suínas, ovinas, bovinas e de aves $12 \%$ (Min.) de proteína, 30\% (Máx.) gorduras totais, 1 - 10\% (Max.) carboidratos totais, 5,0\% (Max.) para amido e 0,1 a 0,9\% de teor de cálcio em base seca. Como demonstra na Tabela 2, as mortadelas atendem aos parâmetros estabelecidos. Porém, apesar do regulamento técnico de qualidade e identidade de mortadela (BRASIL, 2000) não citar pescado como matéria-prima, o teor de umidade das formulações ficou acima da legislação (máximo 65\%). Isto está relacionado com o elevado teor de água existente na CMS utilizada na formulação, que por ser de peixe é rica em umidade, podendo variar atingindo valores de até $80 \%$, segundo Bernadino Filho et al. (2019).

De acordo com a Tabela 2, é possível observar que não houve diferença significativa $(\mathrm{p}<0,05)$ entre os teores de cinzas nas formulações avaliadas. Esses teores são superiores aos reportados por Bessa (2014) que avaliou a composição centesimal de salsichas elaborada com CMS de tilápia, encontrando uma variação entre 2,92 e $3,05 \%$. Possivelmente, a maior quantidade de cinzas encontrada nos emulsionados, devese à adição do extrato elaborado do cefalotórax do camarão, que possui um elevado teor de cinzas, conforme relatado por Bernadino Filho et al. (2019).

\begin{tabular}{|c|c|c|c|}
\hline Parâmetros (\%) & $\mathrm{M}_{0,5}$ & $\mathrm{M}_{0,75}$ & $\mathrm{M}_{1}$ \\
\hline Umidade & $67,07 \pm 0,27^{a}$ & $66,78 \pm 0,28^{a b}$ & $66,17 \pm 0,06^{b}$ \\
\hline Cinzas & $3,68 \pm 0,03^{a}$ & $3,68 \pm 0,04^{\mathrm{a}}$ & $3,72 \pm 0,01^{a}$ \\
\hline Proteínas & $13,13 \pm 0,30^{a}$ & $13,11 \pm 0,18^{a}$ & $13,10 \pm 0,21^{a}$ \\
\hline Lipídeos & $14,97 \pm 0,17^{b}$ & $15,32 \pm 0,18^{a b}$ & $15,88 \pm 0,13^{a}$ \\
\hline Carboidratos Totais & $1,15 \pm 0,22^{a}$ & $1,11 \pm 0,12^{a}$ & $1,13 \pm 0,05^{\mathrm{a}}$ \\
\hline Amido & $0,15 \pm 0,02^{a}$ & $0,15 \pm 0,01^{a}$ & $0,15 \pm 0,09^{a}$ \\
\hline Cálcio* & $0,22 \pm 0,02^{a}$ & $0,26 \pm 0,02^{a}$ & $0,25 \pm 0,03^{a}$ \\
\hline lor calórico (Kcal/100g) & $191,85 \pm 0,33^{b}$ & $194,78 \pm 0,38^{a b}$ & $199,22 \pm 0,15^{a}$ \\
\hline
\end{tabular}

Tabela 2. Caracterização química parcial, teor de cálcio e valor energético das mortadelas de tilápia do Nilo com sabor de camarão

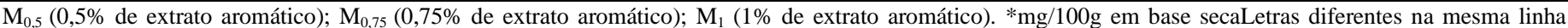
diferem entre si $(\mathrm{p}<0,05)$

Os teores médios de proteína encontrados nas mortadelas variaram de 13,10 a $13,13 \%$, respectivamente para as formulações $\mathbf{M}_{0,75}$ e $\mathbf{M}_{1}$, que foram estatisticamente iguais não diferindo entre si ao nível de 5\% de significância. Avaliando a composição centesimal de salsichas elaboradas com CMS de tilápia do Nilo, Bessa (2014) obtive valores próximos aos encontrados nesta pesquisa. Todavia, nas formulações analisadas, foram encontrados resultados superiores aos teores de proteína mínima preconizados na legislação brasileira para mortadela de carne de aves, bovina e suína, que é de $12 \%$ (BRASIL, 2000).
A formulação $\mathrm{M}_{1}$, que continha uma maior quantidade de extrato de camarão, apresentou o maior teor de lipídeos $(15,88 \%)$ diferindo estatisticamente da formulação M0,5 $(14,97 \%)$, que continha a menor quantidade do extrato. Com estes resultados, pode-se afirmar que a adição de maiores concentrações de extrato de camarão influenciou no aumento do teor de lipídeos nas formulações. Isto pode ser explicado pelo fato do extrato obtido do cefalotórax de camarão apresentar um elevado teor de lipídeos de 12,04\%, segundo Bernadino Filho et al. (2019). 
Quanto ao teor de carboidratos totais e de amido das amostras analisadas, não houve diferença significativas entre as formulações. Isto se deve, possivelmente, ao fato de que as mortadelas foram elaboradas com quantidades iguais da principal fonte de carboidratos (proteína texturizada de soja PTS) usada nas formulações. Valores superiores aos reportados na Tabela 2 para carboidratos totais e amido foram encontrados por Dallabona et al. (2013), ao determinarem a composição centesimal de emulsionados elaborados com o mesmo tipo de matéria-prima desta pesquisa, obtendo $3,51 \%$.

As formulações analisadas apresentaram uma variação de 0,22 a $0,26 \mathrm{mg} / 100 \mathrm{~g}$ de cálcio em base seca, não diferindo estatisticamente entre si ao nível de $5 \%$ de significância. A determinação do teor de cálcio em produtos cárneos emulsionados como a mortadela é importante para se ter uma noção da qualidade da matéria-prima utilizada. Segundo Bernadino Filho et al. (2019), o teor de cálcio em CMS representa uma forma de se controlar os rendimentos obtidos nos processos de separação mecânica, onde maior teor de ossos implica num maior teor de cálcio na matéria-prima.

Quanto aos valores energéticos, a formulação $\mathrm{M}_{1}$ foi a que obteve o teor mais alto $(191,85 \mathrm{Kcal} / 100 \mathrm{~g})$, provavelmente devido a maior adição de extrato aromático, que por sua vez, continha um elevado teor de gordura que é considerada substância altamente energética. Não houve diferença significativa entre as formulações $\mathrm{M}_{0,5}$ e $\mathrm{M}_{0,75}$.

\section{Análises físicas}

De acordo com os resultados obtidos na análise de $\mathrm{pH}$ (Tabela 3), verifica-se que as formulações não diferiram estatisticamente entre si. De acordo com Lourenço et al. (2012), valores de $\mathrm{pH}$ próximos do neutro afeta as proteínas miofibrilares que atingem sua capacidade emulsificante máxima, favorecendo a formação da emulsão, contribuindo para uma textura melhor do produto emulsionado.

Tabela 3. pH, TBARS, Aw, força de cisalhamento, cor e rendimento de cozimento das mortadelas com sabor camarão

\begin{tabular}{cccc}
\hline Análises & $\mathrm{M}_{0,5}$ & $\mathrm{M}_{0,75}$ & $\mathrm{M}_{1}$ \\
\hline $\mathrm{pH}$ & $6,61 \pm 0,01^{\mathrm{a}}$ & $6,63 \pm 0,02^{\mathrm{a}}$ & $6,60 \pm 0,01^{\mathrm{a}}$ \\
TBARS $(\mathrm{mg} / \mathrm{Kg})$ & $0,98 \pm 0,20^{\mathrm{a}}$ & $0,99 \pm 0,18^{\mathrm{a}}$ & $0,98 \pm 0,27^{\mathrm{a}}$ \\
Aw & $0,982 \pm 0,00^{\mathrm{a}}$ & $0,980 \pm 0,00^{\mathrm{a}}$ & $0,983 \pm 0,00^{\mathrm{a}}$ \\
Força de Cisalhamento $\left(\mathrm{Kgf} / \mathrm{cm}^{2}\right)^{*}$ & $0,30 \pm 0,00^{\mathrm{a}}$ & $0,30 \pm 0,00^{\mathrm{a}}$ & $0,30 \pm 0,00^{\mathrm{a}}$ \\
\hline Cor & & & \\
\hline $\mathrm{L}^{*}$ & $61,05 \pm 0,05^{\mathrm{a}}$ & $57,35 \pm 0,55^{\mathrm{b}}$ & $52,45 \pm 0,65^{\mathrm{c}}$ \\
$\mathrm{a}^{*}$ & $7,67 \pm 0,21^{\mathrm{c}}$ & $10,00 \pm 0,14^{\mathrm{b}}$ & $12,70 \pm 0,24^{\mathrm{a}}$ \\
$\mathrm{b}^{*}$ & $18,90 \pm 0,15^{\mathrm{c}}$ & $19,40 \pm 0,15^{\mathrm{b}}$ & $21,15 \pm 0,55^{\mathrm{a}}$ \\
\hline Rendimento de Cozimento (\%) & $95,30 \pm 0,04^{\mathrm{a}}$ & $95,33 \pm 0,03^{\mathrm{a}}$ & $95,31 \pm 0,06^{\mathrm{a}}$ \\
\hline
\end{tabular}

* Kgf = Unidade de medida (quilograma-força). Letras diferentes na mesma linha diferem significativamente pelo teste de Tukey ao nível de $5 \%$ de significância.

Os valores de TBARS (malonaldeído/kg) das amostras não apresentaram diferença significativa entre as formulações desenvolvidas. Segundo Bilgin et al. (2008), o índice de TBARS é utilizado como um indicador do grau de oxidação lipídica, sendo esta responsável por uma redução na qualidade nutricional, bem como alterações no sabor, que podem comprometer a aceitação do produto final. A legislação brasileira não indica um valor máximo permitido de TBARS para produtos de pescado. No entanto, em produtos muito oxidados, ou seja, com valores altos de TBARS, pode haver a formação de compostos tóxicos e cancerígenos (SUMMO et al., 2006). Os valores encontrados nesta pesquisa (Tabela 3) estão de acordo com o valor recomendado para o bom estado de conservação, com relação às alterações oxidativas em produtos cárneos que é de menos de $3 \mathrm{mg} / \mathrm{kg}$ (AL-KAHTANI, 1996).

Os valores de atividade de água (Aw) de todos as formulações foram constantes $(0,98)$, sendo classificadas como alimento de alta atividade de água, o que pode favorecer a proliferação de micro-organismos, necessitando que esse tipo de produto seja armazenado sob refrigeração. Bartolomeu et al. (2014), encontraram valores de Aw de 0,98 em mortadelas elaboradas com o mesmo tipo de matéria-prima usada nesta pesquisa, portanto, estes resultados (Tabela 3 ) estão de acordo com os reportados na literatura.

Para a força de cisalhamento, observou-se que não houve diferença significativa entre todas as formulações, indicando assim que, a adição do extrato em concentrações diferentes não alterou a textura do produto.

Houve diferenças significativas para os parâmetros luminosidade $\left(\mathrm{L}^{*}\right)$, cor vermelha $\left(\mathrm{a}^{*}\right)$ e cor amarela $\left(\mathrm{b}^{*}\right)$ entre todas as formulações. Pode-se observar (Tabela 3 ) que a luminosidade das mortadelas diminuiu à medida que o percentual de extrato aumentou nas formulações. A amostra $\mathrm{M}_{0,5}$ apresentou-se mais clara com a maior média para $\mathrm{o}$ parâmetro $L^{*}(61,05)$, seguida das amostras $M_{0,75}(57,35)$ e $\mathbf{M}_{1}$ $(52,45)$. De acordo com Minozzo $(2010)$, o valor L* expressa a luminosidade ou claridade da amostra, e varia de 0 a 100; assim sendo, quanto mais próximo de 100 , mais clara é a amostra e quanto mais distante, mais escura.

Para todas as formulações os valores de $a^{*}$ e $b^{*}$ foram positivos, onde é possível afirmar que as formulações tenderam às cores vermelha clara e amarela, com tendência a cor alaranjada. As variações nas concentrações de extrato aromático influenciaram estes parâmetros, pois à medida que aumentou a concentração de extrato nas formulações, houve um aumento dos valores de $a^{*}$ e b*. Rizzo e Mauratore (2009) afirmam que a cor dos alimentos é um parâmetro que deve ser levado em consideração, sofrendo diretamente o impacto na aceitabilidade inicial por parte dos consumidores, podendo ser considerada uma das muitas características que definem a compra e o consumo regular do produto. 
Não houve diferença significativa entre o rendimento de cozimento das formulações desenvolvidas. A forma de cozimento, o tipo de envoltório e a composição da matériaprima influenciam no rendimento de cozimento, pois segundo Souza et al. (2005), a gordura também pode influenciar as perdas durante o processo de cocção. Peixes e derivados com maior teor de gordura perdem menos água. A CMS utilizada como matéria-prima neste estudo continha uma quantidade razoável de gordura $(10,63 \%)$, o que pode ter contribuído para um maior rendimento do produto (BERNADINO FILHO; XAVIER, 2019).

\section{CONCLUSÕES}

Todas as formulações de mortadelas apresentaram composição química parcial dentro dos padrões estabelecidos pela legislação vigente no Brasil. As análises de $\mathrm{pH}$ e oxidação lipídica atenderam aos padrões de qualidade recomendados na literatura, comprovando a boa qualidade dos produtos elaborados. Desta forma, as formulações desenvolvidas são uma alternativa para agregar valor aos resíduos do processamento do camarão e da filetagem de tilápia, podendo ser empregados na elaboração de um produto popularmente conhecido, como a mortadela, com características mais saudáveis e nutritivas, podendo ser uma opção para incentivar o consumo de peixe pela população e reduzir a contaminação do meio ambiente ao evitar o descarte desses resíduos de forma inadequada.

\section{REFERÊNCIAS}

AL-KAHTANI, H. A. Chemical changes after irradiation and postirradiation storage in tilapia and Spanish mackerel. Journal of Food Science, v.61, n.4, p. 729-733, 1996. 10.1111/j.13652621.1996.tb12191.

AOAC. Association of Official Analytical Chemists. Official Methods of Analysis, 18ed. Washington. Gaithersburg. 2010. 194p.

BARROSO R. M; PINCINATO R. B. M; MUNOZ A. E. P. Informativo de Mercado da Tilápia: $\mathrm{O}$ mercado da tilápia $-2^{\circ}$ trimestre de 2017. Embrapa Pesca Aquicultura. Palmas, TO. 2017. 1 p.

BARTOLOMEU, D. A. F. S.; WASZCZYNSKYJ N.; KIRSCHNIK P. G.; DALLABONA B. F.; COSTA F. J. O. G.; LEIVAS C. L. Storage of vaccun-packaged smoked bolongna sausage prapared from Nile tilápia. Acta Scientiarum Technology, v.36, n. 3, p. 561-567, 2014. 10.4025/actascitechnol.v36i3.1826.

BASILIO, F. F. F, OGAWA, M., PERDIGÃO, N. B., VASCONCELOS, F. C. Elaboração de saborizante líquido e em pó de cabeça de camarão. In: XIII CONBEP, 2003, Porto Seguro. Resumos... 2003.

BERNADINO FILHO R.; SILVA O. S.; NAVARRO L.A. O; QUEIROGA A. X. M. Q.; OLIVEIRA S. N. O.; OLIVEIRA N.
M.; VASCONCELOS U. A. A. Elaboration of Aromatic Extracts From the Industrial Waste of White Shrimp. Journal of Agricultural Science; v. 11, n. 6, p. 218-224. 2019. $\underline{10.5539 / \mathrm{jas} . v 11 \mathrm{n} 6 \mathrm{p} 218 .}$.

BERNADINO FILHO R.; XAVIER L. C. Obtenção, rendimento e caracterização de $\mathrm{cms}$ produzida com resíduos da filetagem de tilápia do Nilo. Revista Brasileira de $\begin{array}{llllll}\text { Agrotecnologia. } & \text { v. } 09, & \text { v.2, p. } & \text { 01-04. } 2019 .\end{array}$ 10.18378/rebagro.v9i2.7534.

BESSA D. P. Elaboração de Salsicha Prebiótica com Resíduo de Tilápia (Oreochromis niloticus) e Redução de Sódio. 2014, 78f. Dissertação. (Mestrado em Medicina Veterinária) Universidade Federal Fluminense, Nitéroi, 2014.

BILGIN, F.; ÜNLÜSAYIN, M.; İZCI, L.; GÜNLÜ, A. The determination of the shelf life and some nutritional components of gilthead seabream (Sparus aurata L., 1758) after cold and hot smoking. Turkish Journal of Veterinary and Animal Sciences, v. 32, n. 1, p.49-56, 2008.

BRASIL. Agencia Nacional de Vigilância Sanitária (ANVISA). Resolução RDC no 360 de 23 de dezembro de 2003 - Aprova Regulamento Técnico sobre rotulagem nutricional de alimentos embalados, tornando obrigatória a rotulagem nutricional. Diário Oficial da União; Poder Executivo, 2000.

BRASIL. Ministério da Agricultura, Pecuária e do Abastecimento. Regulamento técnico para fixação de identidade e qualidade de carne mecanicamente separada (CMS) de aves, bovinos e suínos. Instrução Normativa $\mathrm{n}^{\circ} 4$ de 31 mar. 2000. Diário Oficial da União; Poder Executivo, 2000.

BUTTE, N.F; CABALLERO, B. Energy needs: Assesssment and Requirements. In: Modern Nutrition in Health and Disease, Maurice Shils et al. (eds.), 10ed. Philadelphia, PA. (USA): Lippincott Williams \& Wilkins. 2006. 2069p.

DALLABONA B. R.; KARAM L. B.; WAGNER R; BARTOLOMEU D. A. F. S.; MIKOS J. D.; FRANSCISCO J. G. P.; MACEDO R. E; KIRSCHNIK P. G. Effect of heat treatment and packaging systems on the stability of fish sausage. Revista Brasileira de Zootecnia. v.42, n.12, p. 835-843, 2013. 10.1590/S1516-35982013001200001.

FOLCH, J.; LESS, M.; STANLEY, S. A simple method for the isolation and purification of total lipids from animal tissues. Journal of Biological Chemistry, v. 226, p. 497-509, 1957.

GONÇALVES A. G. Tecnologia do pescado: ciência, tecnologia, inovação e legislação. 1ed. São Paulo/SP. Atheneu, 2011. 624p.

HORITA, C. N.; MORGANO, M. A.; CELEGHINI, R. M. S.; POLLONIO, M. A. R. Physico-chemical and sensory properties of reduced-fat mortadella prepared with blends of calcium, magnesium and potassium chloride as partial substitutes for 
sodium chloride. Meat Science, Barking, v. 89, n. 4, p. 426-433, 2011. 10.1016/j.meatsci.2011.05.010.

IBGE - Instituto Brasileiro De Geografia e Estatística/ 2016.

LOURENÇO, L. F. H.; GALVÃO, J. C. S.; RIBEIRO, S. C. A.; RIBEIRO, C. F. A.; PARK, K. J. Fat substitutes in processing of sausages using piramutaba waste. Journal of Food Science and Technology, v.01 p.01-09, 2012. 10.1007/s13197-0120645-8.

MINOZZO M. G. Patê De Pescado: Alternativa Para Incremento Da Produção Nas Indústrias Pesqueiras. 2010, $228 \mathrm{f}$. Tese (Doutorado em Tecnologia de Alimentos) - Universidade Federal do Paraná, Curitiba, 2010.

MOREIRA, R. T; LEMOS A. L. S. C; HARADA, M. M; CIPOLLI, K; MENDES, E. S; GUIMARÃES, J. L; CRISTIANINI, M. Desenvolvimento e aceitação de embutido emulsionado tipo mortadela elaborado com tilápia (Oreochromis niloticus L.). Higiene Alimentar, v. 22, n.159, p. 47-52, 2008.

RIZZO, V.; MURATORE, G. Effects of packaging on shelf life os fresh celery. Journal of Food Engineering, v. 90, p. 124-128, 2009. 10.1016/j.jfoodeng.2008.06.011.

SILVA, F. DE A. S. E.; AZEVEDO, C. A. V. The Assistat Software Version 7.7 and its use in the analysis of experimental data. African Journal of Agricultural Science, v.11, n.39, p. 3733-3740, 2016. 10.5897/AJAR2016.11522.

SOUZA, M. L. R.; VIEGAS, E. M. M.; SOBRAL, P. J. A.; KRONKA, S. N. Efeito do peso de tilápia do Nilo (Oreochromis niloticus) sobre o rendimento e a qualidade de seus filés defumados com e sem pele. Ciência e Tecnologia de Alimentos. Campinas, v. 25, n.01, p. 51-59, 2005. 10.1590/S0101-20612005000100009.

SUMMO, C.; CAPONIO, F.; PASQUALONE, A. Effect of vacuum-packaging storage on the quality level of ripened sausages. Meat Science, v.74, p. 249-254, 2006. $\underline{10.1016 / \text { j.meatsci.2006.03.008. }}$.

VYNCKE, W. Direct determination of the thiobarbituric acid value in thichloracetic acid extracts of fish as a measure of oxidative rancidity. Fette Seifen Anstrichmittel, v. 72, n. 12, p. 1084-1087, 1970. 10.1002/lipi.19700721218. 